\title{
Decision support system design for sorting of sheet metal cutting residues
}

\author{
Aleksey Sukhonschikov ${ }^{1}$, and Anatolii Ulitin $^{1}$ \\ ${ }^{1}$ Department of Automation and Computer Engineering, Vologda State University, 160000, Vologda, 15 Lenina st., Russian Federation
}

\begin{abstract}
This article proposes the structure of software package with decision support system elements for managing material resources of sheet metal. Features of interaction between systems that make up the software complex were defined. The system is presented in a form of a set-theoretical description, based on which tasks and connections of modules are determined.
\end{abstract}

\section{Introduction}

At machine-building enterprises, rational usage of sheet metal material resources affects technological costs and other technical and economic indicators of operating activities of the enterprise. In order to increase the utilization factor of sheet metal at enterprises where a significant share of material costs are the costs of sheet metal, rational cutting methods are used and there is a tendency to use cutting residues in subsequent technological processes.

Many works of the Russian and international researchers consider the issues of rational cutting, namely: L.V. Kantorovich, V.A. Zalgaller, E.A. Mukhacheva, V.M. Kartak, A.A. Petunin et al. A special scientific group ESICUP (Euro Special Interest Group on Cutting and Packing) is solving the task of appropriate cutting and packing, and it holds annual international conferences. Most of the currently developed rational cutting methods are based on the use of simple single-pass and multi-pass heuristics, as well as metaheuristic algorithms, such as a genetic algorithm or the tabu search algorithm. There are no analytical methods for solving cutting-packing problems as there does not exist the analytical form for the conditions of mutual non-intersection and location of products on cutting map taking into account the technological limitations [1-3]. Many of CAD/CAM systems allow one to save cutting residue in the database in order to further use it in technological operations. But the decision about whether this cutting residue is usable or not is made by cutting specialist, based on his own empirical perception. Thus, the existing CAD/CAM systems do not implement decision support system (DSS) in the field of sorting the cutting residue material resources into groups of surplus and refuse. The relevance of DSS development is confirmed by works [2,3].

The use of returnable residues, as a rule, creates a number of difficulties at production, as it requires additional costs: residues must be marked, stored, accounted and searched. In addition, when sorting for surplus and refuse, it is necessary to consider the statistical experience in subsequent technological operations usage and to forecast demand for cutting residues. $\mathrm{CAD} / \mathrm{CAM}$ systems do not possess enough information for a reasonable residues sorting. Therefore, it is advisable to build a software package based on interoperation between $\mathrm{CAD} / \mathrm{CAM}$ system and the enterprise accounting system, which belongs to the class of ERP systems, to increase the utilization factor of sheet metal. This implementation of CAD/CAM software package leads to rational cutting, and ERP system rationalizes sorting of cutting residues based on actual operational and statistical information. ERP systems have the necessary statistical information and analytical capabilities to build DSS for sorting of sheet metal cutting residues. The general block diagram of the software package is shown in Figure 1.

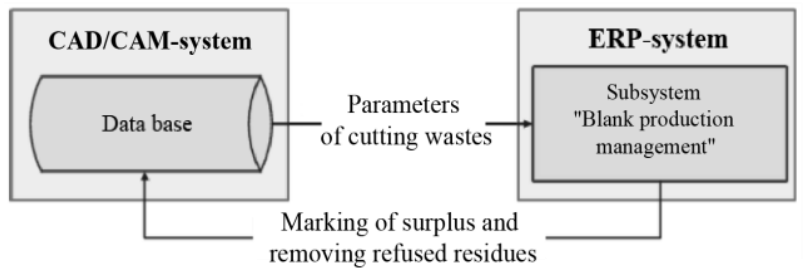

Fig. 1. Block diagram of the software package.

The interaction of CAD/CAM and ERP-systems allows increasing the economic efficiency of materials usage and provides a correct assessment of technological costs.

The "Blank production management" subsystem is constructed from interacting software modules. Such an implementation facilitates integration of this subsystem into the ERP system due to minimal changes in the typical mechanisms of ERP system. Further we describe the modular model of "Blank production management" subsystem, identify tasks of modules, and determine the essence of information exchange between them. Design 
of the subsystem is presented in a form of a settheoretical description that displays the interaction of modules with each other, external software and user. To compile a set-theoretical description, the following basic components are distinguished [4,5] in the subsystem "Blank production management": a set of organizational units - modules; a set of tasks; an environment that represents some space in which modules exist; a set of relationships between modules; a set of module actions.

The presented components allow one to determine the "Blank production management" subsystem as follows:

$$
S I S=\langle M, R, T, Z, Y\rangle,
$$

where $M$ is the set of elements that make up subsystem, in other words, software modules, $R$ is the set of relationships in the set of elements, $T$ is the set of tasks, $Z$ is the input set, $Y$ is the output set.

The following modules are included in the "Blank production management" subsystem: module of system synchronization, DSS module, module of cutting residue accounting, module of scheduled tasks, data base, user interface.

Thus, the set of "Blank production management" subsystem modules is defined by:

$$
M=\left\langle M_{\mathrm{syn}}, M_{\mathrm{dss}}, M_{a}, M_{t}, M_{d b}, M_{\mathrm{int}}\right\rangle,
$$

where $M_{s y n}$ is the module of system synchronization, $M_{d s s}$ is the DSS module, $M_{a}$ is the module of cutting residue accounting, $M_{t}$ is the scheduled tasks module, $M_{d b}$ is the subsystem data base, $M_{\text {int }}$ is the user interface.

Relations between modules are determined by the following set:

$$
R=\left\langle R_{0}, R_{1}, R_{2}, R_{3}, R_{4}, R_{5}, R_{6}, R_{7}, R_{8}, R_{9}, R_{10}\right\rangle,
$$

To show connections between modules, expression 3 can be represented in a tabular form, Table 1.

Table 1. Relations between modules.

\begin{tabular}{|l|l|l|l|l|l|l|}
\hline & $M_{\text {syn }}$ & $M_{d s s}$ & $M_{a}$ & $M_{t}$ & $M_{d b}$ & $M_{\text {int }}$ \\
\hline$M_{\text {syn }}$ & & $R_{0}$ & $R_{5}$ & & & $R_{6}$ \\
\hline$M_{d s s}$ & $R_{0}$ & & $R_{2}$ & & $R_{1}$ & \\
\hline$M_{a}$ & $R_{5}$ & $R_{2}$ & & $R_{7}$ & $R_{4}$ & $R_{3}$ \\
\hline$M_{t}$ & & & $R_{7}$ & & $R_{8}$ & $R_{9}$ \\
\hline$M_{d b}$ & & $R_{1}$ & $R_{4}$ & $R_{8}$ & & $R_{10}$ \\
\hline$M_{\text {int }}$ & $R_{6}$ & & $R_{3}$ & $R_{9}$ & $R_{10}$ & \\
\hline
\end{tabular}

Further we describe in detail the relations in expression 3:

$R_{0}$ is the relation between the DSS module and the module of system synchronization. Using it the systems synchronization module transfers to the DSS module the values of the sheet metal cutting residues indicators from the $\mathrm{CAD} / \mathrm{CAM}$ system. Based on the transferred values, the DSS module further generates a proposal for sorting the cutting residues into surplus and refuse material resources groups.
$R_{I}$ is the relation between the DSS module and the "Blank production management" subsystem data base. The DSS module receives the necessary information from the data base to form a sorting solution. Before generating a sorting suggestion, the DSS module identifies the returnable cutting residues and assigns this residue to a certain class basing on its parameters. The parameters, which define the class of residue, are contained in the data base in a special directory. After identification, a sorting suggestion is generated based on analysis of the residues class. To formulate a sorting suggestion, the DSS module receives from the data base the required amount of residues by class, the amount of given class residues in the warehouse, the intensity of warehouse replenishment with residues of this class and the lost demand for residues.

$R_{2}$ is the relation between the DSS module and the module of cutting residue accounting. The suggestion for sorting the cutting residues generated by the DSS module is sent to the module of cutting residue accounting to make a final decision on assigning the residues to the group of surplus or refuse material resources. The module of cutting residue accounting generates a document "Cutting residues", in which returnable residues are divided into surplus and refuse. Further accounting of the returnable residues is carried out depending on the group which the residue is assigned on the basis of the generated document.

$R_{3}$ is the relation between the module of cutting residue accounting and the user interface. The DSS module generates only a suggestion for sorting the returnable residues, the final decision on whether the residue is surplus or refuse is made by the software package user. The module of cutting residue accounting generates a document "Cutting residues" based on information received from the DSS module. This document can be adjusted by user. The $R_{3}$ relation implements the interactive mode of the user's work with the "Blank production management" subsystem at the stage of "Cutting residues" document formation.

$R_{4}$ is the relation between the module of cutting residue accounting and the "Blank production management" subsystem data base. After the final decision on sorting the cutting residues is made, the corresponding entries are made to the data base. Based on the "Cutting residues" document, the resulting number of residues sorted by class and the number of the received surplus residues are recorded in the data base.

$R_{5}$ is the relation between the module of cutting residue accounting and the module of system synchronization. The synchronization module copies current changes from the ERP system to the CAD/CAM system. Therefore, after sorting the returnable residues into surplus and refuse ones, it provides marking of surplus and deletion of refused cutting residues in CAD/CAM system. Using the $R_{5}$ relation, the synchronization module receives information from the module of cutting residue accounting about current changes in the ERP system.

$R_{6}$ is the relation between the module of system synchronization and the user interface. Using this relation, the synchronization module sends a user request 
to obtain residues parameters of the current cutting for subsequent sorting of these residues.

$R_{7}$ is the relation between the scheduled tasks module, and the module of cutting residue accounting. The appropriateness of storing some residues may change over time. It comes from comparing changes in net income depending on manufacturing of products from surplus residues and the sale of refuse residues to external organizations. In this case, part of the surplus residues stored in warehouse shall be identified as refuse. Periodic reassessment of stored surplus residues is part of the regulatory scheduled tasks module. After this reassessment, it becomes necessary to synchronize the $\mathrm{CAD} / \mathrm{CAM}$ data bases and ERP systems to remove returnable residues identified as refuse from the CAD/CAM system. Cutting residues that are identified as refuse after reassessment need to be re-accounted, this is performed by the module of cutting residue accounting. Thus, $R_{7}$ relation re-accounts the reassessed residues.

$R_{8}$ is the relation between scheduled tasks module and the "Blank production management" subsystem data base. As was stated above, one of the tasks of the scheduled tasks module is reassessment of cutting residues stored in warehouse. The other task of this module is to calculate the required number of residues of each class based on statistical experience. The scheduled tasks receive data for calculation and reassessment from the data base through the relation $R_{8}$.

$R_{9}$ is the relation between scheduled tasks module and user interface. Scheduled tasks are performed according to a given schedule, but can be performed at the user request. The user request for scheduled tasks is transmitted through the interface using relation $R_{9}$.

$R_{10}$ is the relation between user interface and "Blank production management" subsystem data base. The user needs to analyze blank production operations, which implies the availability of relevant reports in the program interface. The data for building reports comes from the data base to the user interface through the relation $R_{I 0}$

Next, a set of tasks for the "Blank production management" subsystem will be described. The set of tasks is defined as:

$$
T=\left\langle T_{\text {get }}, T_{\text {ind }}, T_{\text {sort }}, T_{\text {doc }}, T_{\text {mark }}, T_{\text {del }}, T_{\text {report }}, T_{Q}, T_{\text {cost }}, T_{\text {save }}\right\rangle,(4)
$$

where $T_{\text {get }}$ is obtaining information on the cutting residues from the $\mathrm{CAD} / \mathrm{CAM}$ system; $T_{\text {ind }}$ is assignment of cutting residues to certain classes; $T_{\text {sort }}$ is sorting of the cutting residues into surplus and refuse; $T_{d o c}$ is generation a document for accounting surplus and refused residues; $T_{\text {mark }}$ is marking of surplus residues; $T_{d e l}$ is deleting refuse residues from $\mathrm{CAD} / \mathrm{CAM}$-system; $T_{\text {report }}$ is reporting for analysis of blank production operations; $T_{Q}$ is routine calculation of requirements for cutting residues of each class; $T_{\text {cost }}$ is reassessment of stored residues; $T_{\text {save }}$ is data saving according to the cutting results.

Expression 4 contains the tasks performed by the subsystem as a whole. A set of individual modules tasks is defined as

$$
T_{M}=\left\langle T_{\mathrm{syn}}, T_{\mathrm{dss}}, T_{\mathrm{a}}, T_{\mathrm{t}}, T_{\mathrm{db}}, T_{\mathrm{int}}\right\rangle,
$$

$T_{s y n}$ is the set of system synchronization tasks:

$$
T_{\text {syn }}=\left\langle T_{\text {get }}, T_{\text {upd }}, T_{\text {give }}\right\rangle,
$$

where $T_{\text {get }}$ is obtaining parameters of cutting residues obtained from CAD/CAM-system, $T_{\text {upd }}$ is renaming of surplus residues in the $\mathrm{CAD} / \mathrm{CAM}$-system in accordance with their class and removing from the CAD/CAMsystem identified identified as refuse, $T_{\text {give }}$ is providing of cutting residues parameters for other subsystem modules.

$T_{d s s}$ is the set of DSS module tasks:

$$
T_{d s s}=\left\langle T_{\text {ind }}, T_{\text {sort }}\right\rangle
$$

where $T_{\text {ind }}$ is the residue identification, implying assignment to a particular class, $T_{\text {sort }}$ is formation of a suggestion for sorting of cutting residues into groups of surplus and refuse material resources.

$T_{a}$ is the set of tasks for the module of cutting residues accounting:

$$
T_{a}=\left\langle T_{\mathrm{doc}}, T_{\text {mark }}, T_{\mathrm{del}}\right\rangle,
$$

where $T_{d o c}$ is the "Cutting residues" document generation with results of identification and sorting of cutting residues for subsequent accounting, $T_{\text {mark }}$ is the surplus residues marking according to their class, $T_{d e l}$ is removing of refused returnable residues from the $\mathrm{CAD} / \mathrm{CAM}$ system

$T_{t}$ is the set of tasks for the scheduled tasks module:

$$
T_{t}=\left\langle T_{\mathrm{Q}}, T_{\cos t}\right\rangle
$$

where $T_{Q}$ is calculation of the need for cutting residues of each class, $T_{\text {cost }}$ is reassessment of stored residues.

$T_{d b}$ is the set of tasks for data base:

$$
T_{d b}=\left\langle T_{\text {save }}, T_{\text {data }}\right\rangle,
$$

where $T_{\text {save }}$ is data saving according to the cutting results, $T_{\text {data }}$ is data providing for reporting and other modules.

$T_{\text {int }}$ is the set of tasks for the user interface:

$$
T_{\mathrm{int}}=\left\langle T_{\text {dialog }}, T_{\text {time }}, T_{\text {rec }}, T_{\text {report }}\right\rangle,
$$

where $T_{\text {dialog }}$ is providing a dialogue mode when deciding on sorting the cutting residues into surplus and refuse, $T_{\text {time }}$ is customization of scheduled tasks, $T_{\text {rec }}$ is recording in data base directories, $T_{\text {report }}$ is report generation.

A set of inputs in the "Blank production management" subsystem is:

$$
Z=\left\langle Z_{\text {info }}, Z_{\text {user }}\right\rangle
$$


where $Z_{\text {info }}$ is information about cutting residues, $Z_{\text {user }}$ is control signals and information from the software package users.

A set of inputs of individual subsystem modules is defined as follows:

$$
Z_{M}=\left\langle Z_{\text {syn }}, Z_{\text {dss }}, Z_{a}, Z_{\mathrm{t}}, Z_{d b}, Z_{\text {int }}\right\rangle
$$

In expression 13:

$Z_{\text {syn }}$ is the set of synchronization module inputs:

$$
Z_{\text {syn }}=\left\langle Z_{\text {res }}, Z_{\text {info }}, Z_{\text {upd }}\right\rangle
$$

where $Z_{\text {res }}$ is request for the receipt of cutting residues from a $\mathrm{CAD} / \mathrm{CAM}$ system and for synchronization of $\mathrm{CAD} / \mathrm{CAM}$ data bases and ERP systems, $Z_{\text {info }}$ is information on returnable cutting residues, $Z_{\text {upd }}$ is request to rename surplus residues in a CAD/CAM system taking into account assigned class and to delete refused residues.

$Z_{d s s}$ is the set of DSS module inputs:

$$
Z_{d s s}=\left\langle Z_{\text {indss }}, Z_{\mathrm{QN}}\right\rangle \text {, }
$$

where $Z_{\text {indss }}$ is information about cutting residues, $Z_{Q N}$ is data from the data base on the current mount of stock residues and the required amount of residue by class.

$Z_{a}$ is the set of inputs for cutting residues module:

$$
Z_{a}=\left\langle Z_{\text {sort }}, Z_{\text {rul }}, Z_{\text {rult }}\right\rangle \text {, }
$$

where $Z_{\text {sort }}$ is the DSS suggestion for sorting of sheet metal cutting residues, $Z_{\text {rul }}$ is the user decision on sorting, $Z_{\text {rult }}$ is the reassessment results for stock residues

$Z_{t}$ is the set of inputs for scheduled tasks module:

$$
Z_{t}=\left\langle Z_{\text {start }}, Z_{\mathrm{dbt}}\right\rangle
$$

where $Z_{\text {start }}$ is settings for scheduled task execution, $Z_{d b t}$ is information from data base

$Z_{d b}$ is the set of inputs for data base:

$$
Z_{d b}=\left\langle Z_{\text {cat }}, Z_{\text {rec }}, Z_{Q}, Z_{r e p}\right\rangle,
$$

where $Z_{\text {cat }}$ is handbook filling, $Z_{\text {rec }}$ is records on accounting registers of the cutting residues, $Z_{Q}$ is the required residues amount by classes, $Z_{\text {rep }}$ is request for generating a report.

$Z_{\text {int }}$ is the set of inputs of user interface:

$$
Z_{\text {int }}=\left\langle Z_{\text {query }}, Z_{\text {report }}, Z_{\text {user }}\right\rangle
$$

where $Z_{\text {query }}$ is suggestion on sorting the cutting residues, $Z_{\text {report }}$ is data from the data base for generating a report, $Z_{\text {user }}$ is control signals and user input information.

A set of outputs in the "Blank production management" subsystem is:

$$
Y=\left\langle Y_{\text {info }}, Y_{\text {upds }}, Y_{E R P}, Y_{\text {user }}\right\rangle,
$$

where $Y_{\text {info }}$ is a request to $\mathrm{CAD} / \mathrm{CAM}$-system for obtaining the cutting residues parameters, $Y_{u p d s}$ is a request to $\mathrm{CAD} / \mathrm{CAM}$-system for renaming surplus residues and deleting refuse, $Y_{E R P}$ is results of sorting of cutting residues for postings according to the standard registers of the ERP system, $Y_{\text {user }}$ is the question for user and output of the subsystem results.

A set of outputs of individual subsystem modules is defined as follows:

$$
Y_{M}=\left\langle Y_{\mathrm{syn}}, Y_{\mathrm{dss}}, Y_{a}, Y_{\mathrm{t}}, Y_{d b}, Y_{\mathrm{int}}\right\rangle
$$

In expression 21:

$Y_{\text {syn }}$ is a set of outputs for synchronization system module:

$$
Y_{s y n}=\left\langle\mathrm{Y}_{\text {info }}, \mathrm{Y}_{\text {upds }}, Y_{\text {indss }}\right\rangle
$$

where $Y_{\text {info }}$ is request to $\mathrm{CAD} / \mathrm{CAM}$ system to receive information on returnable cutting residues, $Y_{u p d s}$ is request to $\mathrm{CAD} / \mathrm{CAM}$ system to rename surplus residues and to delete refused residues, $Y_{\text {indss }}$ is the cutting residues parameters.

$Y_{d s s}$ is the set of output of DSS module:

$$
Y_{d s s}=\left\langle Y_{\text {sort }}\right\rangle \text {, }
$$

where $Y_{\text {sort }}$ is a suggestion for sheet metal cutting residues sorting.

$Y_{a}$ is the set of inputs of cutting residues module:

$$
Y_{a}=\left\langle Y_{r e c}, Y_{\mathrm{ERP}}, Y_{\text {upd }}, Y_{\text {query }}\right\rangle,
$$

where $Y_{\text {rec }}$ is postings on the registers of the data base of the "Blank production management" subsystem, $Y_{E R P}$ is postings on typical ERP system registers, $Y_{u p d}$ is request to rename surplus residues in a CAD/CAM system taking into account the assigned class and to delete refused residues, $Y_{\text {query }}$ is a suggestion for sorting of cutting residues.

$Y_{t}$ is the set of outputs of the scheduled tasks:

$$
Y_{t}=\left\langle Y_{\mathrm{Q}}, Y_{\text {rult }}\right\rangle
$$

where $Y_{Q}$ is the required amount of residues by classes, $Y_{\text {rult }}$ is the reassessment results for the stored residues.

$Y_{d b}$ is the set of output of data base:

$$
Y_{d b}=\left\langle Y_{\mathrm{dbt}}, Y_{\text {report }}, Y_{Q N}\right\rangle
$$

where $Y_{d b t}$ is the statistics from the data base, $Y_{\text {report }}$ is the reporting data, $Y_{Q N}$ is the data on the current number of stock residues and the required amount of residues by classes.

$Y_{\text {int }}$ is the set of output of user interface:

$$
Y_{\text {int }}=\left\langle Y_{\text {res }}, Y_{\text {rul }}, Y_{\text {start }}, Y_{\text {cat }}, Y_{\text {user }}, Y_{\text {rep }}\right\rangle \text {, }
$$




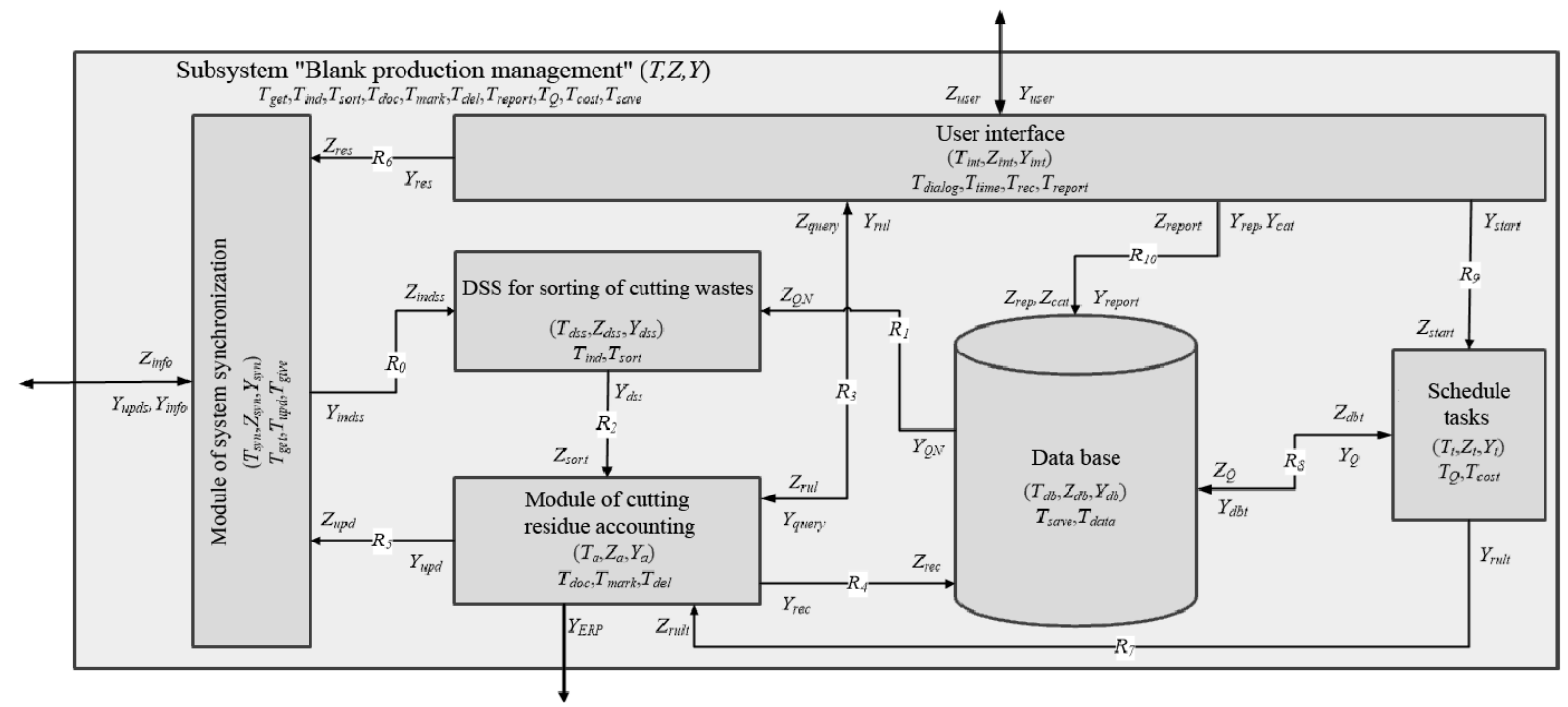

Fig. 2. Block diagram of the "Blank production management" subsystem.

where $Y_{\text {res }}$ is request for receipt of cutting residues from the $\mathrm{CAD} / \mathrm{CAM}$ system, $Y_{\text {rul }}$ is the user solution for sorting cutting residues into surplus and refuse, $Y_{\text {start }}$ is settings of the scheduled task execution, $Y_{\text {cat }}$ is handbook filling, $Y_{\text {user }}$ is the question for user and output of the subsystem results, $Y_{\text {rep }}$ is the reporting request

Thus, the structure of the "Blank production management" subsystem can be represented as follows (Fig. 2).

The following results were achieved at this stage of the study:

- The structure of software package for managing material resources of sheet metal with DSS elements is proposed;

- Features of interaction of software package systems are defined;

- The design of the "Blank production management" subsystem is presented in a form of a set-theoretical description;

- The tasks and connections of modules, which make up the subsystem, are specified.

\section{References}

[1] Yu.I. Valiakhmetova, A.S. Filippova, The L. V. Kantorovich theory of optimal use of resources in cutting-packing problems: a review and history of the development of solution methods, Bulletin of the Ufa State Aviation Technical University, 1, 186-197 (2014).

[2] A.M. Zadorozhny, A.V. Chuvakov, Positive aspects of DSS usage, work principles and classification, Theory and practice of modern science, 6, 24, 306-309 (2017).

[3] V.M. Kureichik, I.B. Safronenkova, Development of DSS architecture for choosing methods for solving layout problems, Information Technologies, 10, 23, 42-48 (2017).

[4] V.B. Tarasov, System-organizational approach in artificial intelligence, Software products and systems, 3, 6-13 (1997).

[5] V.B. Tarasov, Evolutionary semiotics and fuzzy multi-agent systems as the main theoretical approaches to construction of intelligent organizations, Information technologies and computing systems, 1, 54-68 (1998). 